\title{
Positive staining for cellulose in oral pulse granuloma
}

\section{Virkkunen, Sirke}

2017-04

Virkkunen, S, Wolff, H, Haglund, C , Hojgaard, C, Winther , J R, Willemoes , M , Vogel , U \& Hagstrom , J 2017 , ' Positive staining for cellulose in oral pulse granuloma ' , Oral surgery, oral medicine, oral pathology and oral radiology , vol. 123 , no. 4 , pp. 464-467 . https://doi.org/10.1016/j.oo

http://hdl.handle.net/10138/236751

https://doi.org/10.1016/j.000o.2016.11.022

publishedVersion

Downloaded from Helda, University of Helsinki institutional repository.

This is an electronic reprint of the original article.

This reprint may differ from the original in pagination and typographic detail.

Please cite the original version. 


\title{
Positive staining for cellulose in oral pulse granuloma
}

\author{
Sirke Virkkunen, BD, ${ }^{\text {a }}$ Henrik Wolff, MD, PhD, ${ }^{\mathrm{b}}$ Caj Haglund, MD, PhD, ${ }^{\mathrm{c}, \mathrm{d}}$ Casper Højgaard, MSc, \\ Jakob Rahr Winther, PhD, ${ }^{e}$ Martin Willemoës, PhD, ${ }^{e}$ Ulla Vogel, PhD, ${ }^{f}$ and Jaana Hagström, DDS, PhD ${ }^{g}$
}

\begin{abstract}
Objective. Oral pulse granuloma (OPG) is an oral inflammatory lesion characterized by the presence of hyaline rings with numerous multinucleated giant cells. The etiopathogenesis of this lesion is thus far unclear, as is the composition of the hyaline rings. Our aim was to investigate whether the hyaline rings contain cellulose.

Study Design. Using a newly developed staining method for cellulose, we studied 18 histologic samples diagnosed as OPG, in addition to 3 samples originally diagnosed as "normal" foreign body reactions. In our study, visualization of cellulose is based on its specific binding to the carbohydrate binding module of $\beta$-1,4-glycanase.

Results. All samples diagnosed as OPG were positive for cellulose staining localized in hyaline rings. In addition, 1 lesion (of 3), first diagnosed as a foreign body reaction without the presence of hyaline rings, was positive for cellulose by horseradish peroxidase staining.

Conclusions. We show for the first time that cellulose is present in OPG lesions, indicating that cellulose might be the initial cause of formation of these lesions. (Oral Surg Oral Med Oral Pathol Oral Radiol 2017;123:464-467)
\end{abstract}

In 1971, an unusual giant-cell granulomatous lesion affecting mainly the posterior mandibular ridge, in both the periapical and sulcus areas was first described. ${ }^{1,2}$ This process is also observed in soft tissue and cyst walls and is characterized by the presence of hyaline rings with numerous multinucleated giant cells around and within the rings. The lesion contains areas of fibrosis with mature fibroblasts, vacuolated macrophages, plasma cells, and lymphocytes. ${ }^{1}$ Its etiology and pathogenesis are unclear. ${ }^{1,2}$ This lesion is widely termed oral pulse granuloma (OPG); other names have also been used, including chronic mandibular periostitis, giant cell angiopathy, hyaline bodies and giant cells associated with radicular cyst, PG, oral vegetable granuloma, hyaline ring granuloma, food-induced granuloma, and oral pulse or hyaline ring granuloma. ${ }^{2,3}$

There are 2 main hypotheses for OPG pathogenesis: endogenous and exogenous theories. In the endogenous theory, the hyaline rings in OPG are caused by hyaline degenerative changes in the walls of blood vessels. In the exogenous theory, food particles provoke a foreign

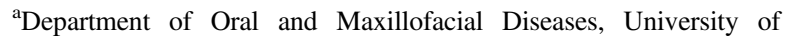
Helsinki and Helsinki University Hospital, Helsinki, Finland.

${ }^{\mathrm{b}}$ Finnish Institute of Occupational Health, Helsinki, Finland.

${ }^{c}$ Department of Surgery, University of Helsinki and Helsinki University Hospital, Helsinki, Finland.

${ }^{\mathrm{d}}$ Research Programs Unit, Translational Cancer Biology, University of Helsinki, Helsinki, Finland.

${ }^{\mathrm{e} S}$ Section for Biomolecular Sciences, Department of Biology, University of Copenhagen, Copenhagen, Denmark.

${ }^{\mathrm{f}}$ National Research Centre for the Working Environment, Copenhagen, Denmark.

${ }^{\mathrm{g}}$ Department of Pathology, Helsinki University and Helsinki University Hospital, Helsinki, Finland.

Received for publication Jul 13, 2016; returned for revision Nov 8, 2016; accepted for publication Nov 29, 2016.

(c) 2016 Elsevier Inc. All rights reserved.

2212-4403/\$ - see front matter

http://dx.doi.org/10.1016/j.00oo.2016.11.022 body reaction due to implantation of cellulosecontaining particles of plant origin, which are visualized as hyaline rings. Implantation occurs in extraction sockets, deep periodontal pockets, unfilled root canals, and grossly decayed teeth. An earlier ultrastructural study suggested that collagen was the fibrillar material in the hyaline rings. ${ }^{4}$ However, as no clear evidence has been found to support either of these hypotheses, it is important to clarify the origin of the hyaline rings. ${ }^{1,5}$

Cellulose may be central to the exogenous theory of OPG pathogenesis. It is one of the most important natural polymers and is used in several industrial sectors. $^{6}$ It is an unbranched homopolysaccharide composed of $\beta$-D-glucopyranose units linked by glycosidic bonds. In nature, cellulose chains have approximately 10,000 glucopyranose units in wood and 15,000 units in cotton. The long chain lengths and the impact of the $\beta$-glycosidic bond on the structure are the main reasons for the insoluble characteristic of cellulose. ${ }^{7}$ Cellulose cannot be digested by mammals without the aid of microbes.

Extremely small cellulose particles, such as nanofibrillar cellulose, are difficult to detect in biological tissues. Knudsen et al. reported that a biotinylated cellulose-binding domain (CBM) of $\beta$-1,4-glycanase (EXG:CBM) of a cellulase enzyme can be used to visualize pulp cellulose as well as 4 different types of nanofibrillar cellulose fibers in biological tissue, and that specific staining can be achieved using horseradish peroxidase (HRP)-tagged avidin labeling.

\section{Statement of Clinical Relevance}

We show for the first time the presence of cellulose in oral pulse granulomas. 
Table I. Summary of cases and results in cellulose staining

\begin{tabular}{llll}
\hline \multicolumn{1}{c}{ Specific localization } & Diagnosis & Previous procedure & Dye result \\
\hline Tooth \#8 apex & OPG & Root canal treatment & Positive \\
Tooth \#8 apex & OPG & Root canal treatment & Positive \\
Tooth \#14 extraction socket & OPG & Extraction & Positive \\
Tooth \#17 follicular space & OPG & Unknown & Positive \\
Tooth \#17 follicular space & OPG & Unknown & Positive \\
Tooth \#17 distal and lateral & OPG & Unknown & Positive \\
side & & & \\
Teeth \#17-18 follicular spaces & OPG & Unknown & Positive \\
Tooth \#18 extraction socket & OPG & Extraction & Positive \\
Tooth \#18 apex & OPG & Root canal treatment & Positive \\
Tooth \#18 distobuccal space & OPG & Unknown & Positive \\
Tooth \#19 apex & OPG & Root canal treatment & Positive \\
Teeth \#21-22 & OPG & Extraction & Positive \\
Tooth \#24 apex & Foreign body & Root canal treatment & Negative \\
Teeth \#24-25 apex & Foreign body & Root canal treatment & Positive \\
Tooth \#25 apex & OPG & Root canal treatment & Positive \\
Tooth \#29 extraction socket & OPG & Extraction & Positive \\
Teeth \#31-32 & OPG & Extraction & Positive \\
Tooth \#32 follicular space & OPG & Unknown & Positive \\
Tooth \#32 follicular space & OPG & Unknown & Positive \\
Ramus & OPG & Extraction & Positive \\
Unknown & Foreign body & Unknown & Negative \\
\hline OPG, 0 . & & &
\end{tabular}

$O P G$, oral pulse granuloma.

\section{MATERIALS AND METHODS \\ Tissue material}

Eighteen tissue samples diagnosed as OPG were collected from the pathology archives of HUSLAB (provider of clinical laboratory services for Helsinki University Central Hospital). In addition, we studied 3 tissue samples from the oral cavity primarily diagnosed as foreign-body reactions (Fig 1). The original diagnoses for these cases were reconfirmed by one of the authors (J.H.). Institutional approval to use the samples was received. The diagnosis, location, previous procedures, and results from the cellulose staining are listed in Table I.

\section{Immunohistochemistry}

For cellulose staining, we used the method described by Knudsen et al. This method is specific and sensitive for detecting cellulose due to a biotinylated CBM of $\beta$-1,4-glycanase (EXC:CBM) from the bacterium Cellulomonas fimi. EXG:CBM, expressed in Escherichia coli, was purified and biotinylated. Biotinylated EXG:CBM was used to visualize cellulose fibers by HRP-tagged avidin labeling, and the HRP-EXG:CBM complex was used to visualize cellulose fibers in tissue slides that were $4 \mu \mathrm{m}$ thick, as previously described. ${ }^{8}$

For EXG:CBM staining, paraffin was removed from the sections. Sections were washed with phosphatebuffered saline (PBS), and endogenous peroxidase was blocked. After washing with PBS, 30\% normal serum with avidin was applied to the sections and then removed with suction. Biotinylated EXG was applied to the sections. After washing in PBS, streptavidinconjugated peroxidase was applied to the sections, and peroxidase enzyme activity was visualized. Sections were then counterstained with Mayer's hematoxylin, dried, and mounted.

\section{Immunohistochemical scoring}

The samples were scored positive if staining was detected. There was negligible background staining in the EXG:CBM system, whereas the staining intensity remained constant for cellulose, allowing the detection of even small amounts of cellulose. However, in general, the amounts of stained material were quite substantial, especially in structures identified as hyaline rings.

\section{RESULTS}

\section{Positive staining}

Nineteen out of 21 samples stained positive for cellulose, including all cases originally diagnosed as OPG (Table I). Positive staining in OPG cases was particularly evident in the hyaline ring structures seen with hematoxylin and eosin (H\&E), but also in other areas.

Of the 3 samples with the diagnosis of a foreign body reaction, 1 was positive and 2 were negative for cellulose. On reevaluation of the positive staining in a sample originally diagnosed as a foreign body reaction, the area of positivity was recognizable as a hyaline ring by $\mathrm{H} \&$ E staining (Table I). 

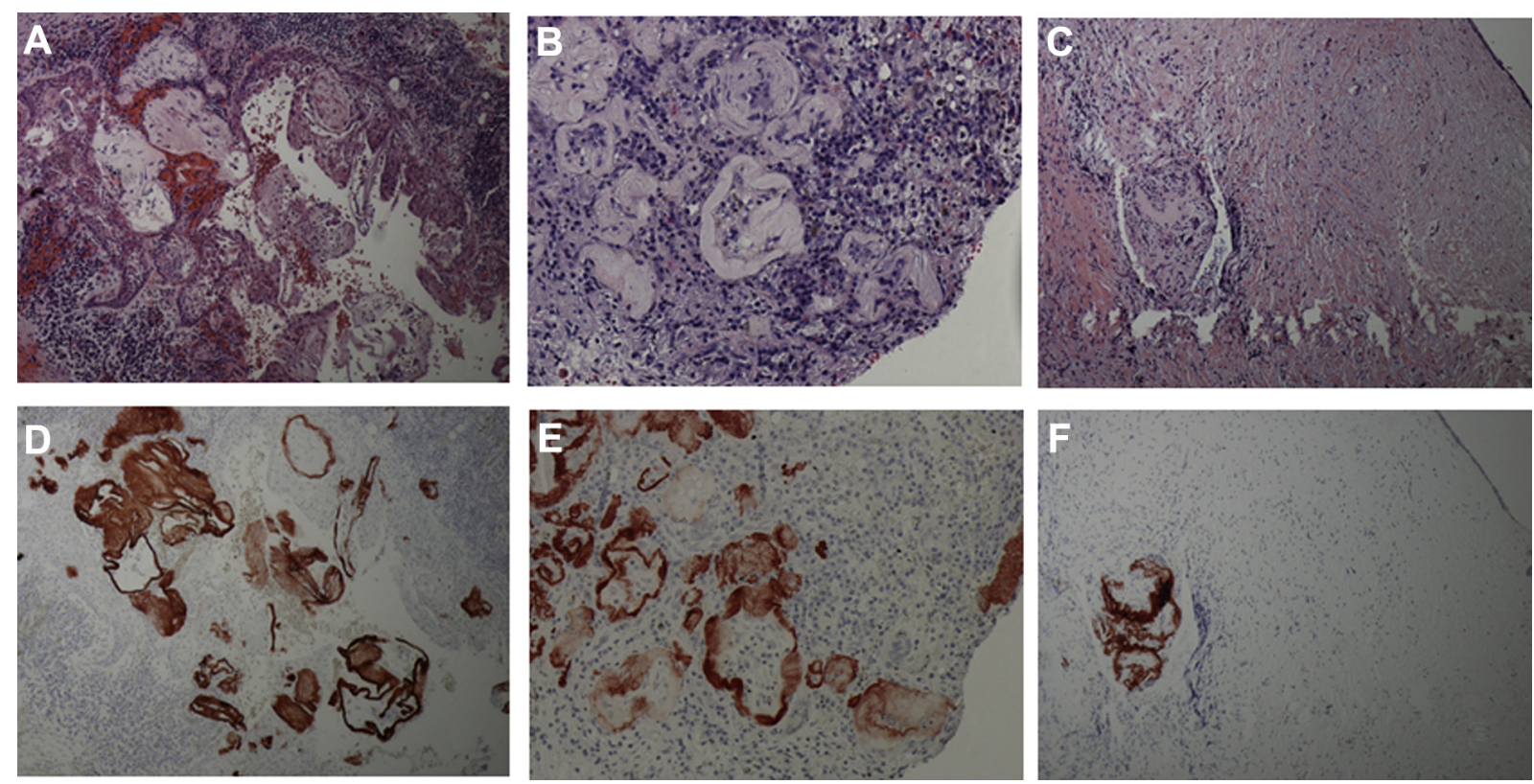

Fig. 1. A-C, Hematoxylin and eosin staining of tissue slides; D-F, the same tissues stained with EXG:carbohydrate binding molecule. In the case of slides B and E, the staining was made from consecutive slides, additionally demonstrating that the hyaline rings stain positively for cellulose. Magnification $\times 200$.

\section{DISCUSSION}

This study represents the first known investigation reporting the presence of cellulose in OPG. We revealed the presence of cellulose in 18 OPG cases, as well as 1 of 3 cases without a prior diagnosis of OPG. Regarding positive cellulose staining in foreign body reactions, hyaline rings could be detected on close examination of H\&Estained materials. This suggests that staining of cellulose could be useful for differentiating OPG from other types of foreign body reactions. Our study challenges the hypothesis that OPG is a result of hyalinization in blood vessels. Using EXG:CBM, we show that the hyaline rings consist of cellulose, which suggests that a foreign body reaction to cellulose could be the principal cause of OPG.

OPG is most commonly found in the mandible, particularly in the posterior regions, where food stagnation is common. ${ }^{1,9}$ Our results suggest that the cellulose moiety of plant-based foods persists in the form of hyaline material, inciting a chronic inflammatory response in the jaw. ${ }^{2}$ Cellulose penetrates tissues through extraction sockets because of stagnation of plant material and seeds. In cases of root-canal treatment, cellulose moieties can enter the jaw via uncovered root canals that provide food particles a pathway to the bone. If the food particles are not the reason for granuloma formation in root apices, it can also be speculated that small pieces of cellulose-containing taper used in root canal treatment could be the cause of these lesions. Indeed, earlier studies in animal models have shown that vegetable material can cause foreign body reactions with lesions similar to OPG. ${ }^{10,11}$
The visualization of cellulose in this study was based on the specific binding of the CBM of $\beta-1,4$-glycanase to cellulose. We have previously shown that EXG:CBM binds with high affinity to different kinds of cellulose, and we have reportd highly specific staining of cellulose in paraffin-embedded tissue. ${ }^{8}$ The assay proved to be both robust and very sensitive, as a small amount of nanocellulose was readily detected after pulmonary exposure in mice. Similarly, the assay is highly specific, and there has been no indication that it stains blood vessel walls or collagen. This work provides strong evidence that these hyaline rings in OPG consist of cellulose and represents the first investigation to use this technique to visualize cellulose in OPG.

\section{CONCLUSION}

Our results are consistent with the hypothesis that the pathognomonic feature of OPG, hyaline rings, consists of cellulose and not of blood vessel walls. Thus, cellulose appears to be the cause of the foreign body reaction seen in OPG.

We thank Jennifer Rowland for helping us with linguistic problems and Sauli Savukoski for laboratory work.

\section{REFERENCES}

1. Philipsen HP, Reichart PA. Pulse or hyaline ring granuloma. Review of the literature on etiopathogenesis of oral and extraoral lesions. Clin Oral Investig. 2010;14:121-128. 
2. Acharya S, Hallikeri K, Anehosur V, et al. Oral pulse or hyaline ring granuloma: a case report and a brief review. J Indian Soc Periodontol. 2015;19:327.

3. Omezli MM, Torul D, Efe Can Sivrikaya EC. The prevalence of foreign bodies in jaw bones on panoramic radiography. Indian $J$ Dent. 2015;6:185.

4. El-Labban NG, Kramer IR. The nature of the hyaline rings in chronic periostitis and other conditions: an ultrastructural study. Oral Surg Oral Med Oral Pathol. 1981;51:509-515.

5. Naik SV, Ghousia S, Shashibhushan K, et al. Pediatric oral pulse granuloma: a rare entity. J Dent Res. 2012;9:812.

6. Liu R, Yu H, Huang Y. Structure and morphology of cellulose in wheat straw. Cellulose. 2005;12:25-34.

7. O'Sullivan AC. Cellulose: the structure slowly unravels. Cellulose. 1997;4:173-207.

8. Knudsen KB, kofoed C, Espersen R, et al. Visualization of nanofibrillar cellulose in biological tissues using a biotinylated carbohydrate binding module of $\beta-1,4$-glycanase. Chem Res Toxicol. 2015;28:1627-1635.
9. Scivetti M, Lucchese A, Ficarra G, et al. Oral pulse granuloma: histological findings by confocal laser scanning microscopy. Ultrastruct Pathol. 2009;33:155-159.

10. Talacko AA, Radden BG. The pathogenesis of oral pulse granuloma: an animal model. J Oral Pathol. 1988;17:99-105.

11. Watson RE, Stewart C. Experimental oral foreign body reactions: vegetable materials. Oral Surg Oral Med Oral Pathol. 1991;71: 312-316.

\section{Reprint requests:}

Jaana Hagström, DDS, PhD

Helsinki University and Helsinki University Hospital Pathology

Haartmaninkatu 3

P.O.BOX 400

00029 HUS

Helsinki 00014

Finland

jaana.hagstrom@hus.fi 